Proceedings of the 8th Polish Symposium of Physics in Economy and Social Sciences FENS, Rzeszów, November 4-6, 2015

\title{
Invariant Value Functions under Cumulative Prospect Theory
}

\author{
S. WÓJCIK* \\ Department of Mathematical Statistics, Statistical Office in Rzeszów, \\ Jana III Sobieskiego 10, 35-959 Rzeszów, Poland
}

\begin{abstract}
In this paper the notion of the preference homogeneity is extended. We determine the value functions under the cumulative prospect theory such that the certainty equivalents related to them are invariant with respect to some classes of transformations.
\end{abstract}

DOI: 10.12693/APhysPolA.129.955

PACS/topics: 89.20.-a, 89.65.Gh

\section{Introduction}

One of the frequently applied normative and descriptive models of decision making under risk is the cumulative prospect theory, developed by Tversky and Kahneman [1]. Under this model the decision maker's preferences over a family $\Delta$ of all lotteries, that is the finitelyvalued random variables defined on a probability space $(\Omega, \Sigma, P)$, are represented by the functional $V: \Delta \rightarrow \mathbb{R}$ of the form

$$
V(X)=E_{g h} u(X) \text { for } X \in \Delta,
$$

where $E_{g h}$ is the generalized Choquet integral with respect to the probability distortion functions $g$ and $h$ for gains and losses, respectively, and $u: \mathbb{R} \rightarrow \mathbb{R}$ is a value function. Let us recall that if $g$ is a probability distortion function, that is a non-decreasing continuous function $g:[0,1] \rightarrow[0,1]$ with $g(0)=0$ and $g(1)=1$ then, for every $X \in L^{\infty}(\Omega, \Sigma, P)$, the Choquet integral is defined in the following way:

$$
\begin{aligned}
& E_{g} X:=\int_{-\infty}^{0}(g(P(X>t))-1) \mathrm{d} t \\
& +\int_{0}^{\infty} g(P(X>t)) \mathrm{d} t .
\end{aligned}
$$

Here $L^{\infty}(\Omega, \Sigma, P)$ stands for the space of all bounded random variables on the probability space $(\Omega, \Sigma, P)$. The generalized Choquet integral is defined as follows:

$$
\begin{aligned}
& E_{g h} X:=E_{g}(\max \{X, 0\})-E_{h}(\max \{-X, 0\}) \\
& \quad \text { for } X \in L^{\infty}(\Omega, \Sigma, P),
\end{aligned}
$$

where $g$ and $h$ are the probability distortion functions for gains and losses, respectively. For every $x_{1}, x_{2} \in \mathbb{R}$ and $p \in[0,1]$, by $\left\langle x_{1}, 1-p ; x_{2}, p\right\rangle$ we denote the random variable taking the values $x_{1}$ and $x_{2}$ with probabilities $1-p$ and $p$, respectively. Furthermore, we set

$$
\mathcal{X}_{2}:=\{\langle 0,1-p ; x, p\rangle: x>0, p \in[0,1]\} .
$$

\footnotetext{
*e-mail: s.wojcik@stat.gov.pl
}

From (2) and (3) we derive that if $X=\left\langle x_{1}, 1-p ; x_{2}, p\right\rangle$ for some $x_{1}, x_{2} \in \mathbb{R}$ and $p \in[0,1]$, then

$$
\begin{aligned}
& E_{g h}(X)=(1-g(p)) x_{1}+g(p) x_{2} \\
& \quad \text { whenever } 0 \leq x_{1}<x_{2},
\end{aligned}
$$

and

$$
\begin{gathered}
E_{g h}(X)=h(1-p) x_{1}+g(p) x_{2} \\
\text { whenever } x_{1}<0<x_{2} .
\end{gathered}
$$

In what follows we assume that the value function $u$ : $\mathbb{R} \rightarrow \mathbb{R}$ is strictly increasing, continuous and $u(0)=0$. Moreover, as the generalized Choquet integral is positively homogeneous, it is reasonable to assume that $u(1)=1$. According to the assumed properties of $u$ it is not difficult to check that for every $X \in \Delta$ there exists exactly one $C_{u}(X) \in \mathbb{R}$ such that $V(X)=u\left(C_{u}(X)\right)$. Therefore, the last equation defines a functional $C: \Delta \rightarrow$ $\mathbb{R}$, called the certainty equivalent. In view of (1), we get

$$
C_{u}(X)=u^{-1}\left(E_{g h} u(X)\right) \text { for } X \in \Delta .
$$

If $\lambda \in(0, \infty)$, then the certainty equivalent is said to be $\lambda$-homogeneous on a family $\mathcal{X} \subset \Delta$, provided $C_{u}(\lambda X)=\lambda C_{u}(X)$ for $X \in \mathcal{X}$. The certainty equivalent is said to be positively homogeneous on $\mathcal{X}$, provided it is $\lambda$-homogeneous on $\mathcal{X}$ for every $\lambda \in(0, \infty)$. Tversky and Kahneman [1] stated, without a proof, that the certainty equivalent is positively homogeneous on $\mathcal{X}_{2}$ if and only if the value function is of the form

$$
u(x)= \begin{cases}x^{\alpha} & \text { for } x \geq 0, \\ -\kappa(-x)^{\beta} & \text { for } x<0\end{cases}
$$

with some $\alpha, \beta, \kappa>0$. A formal proof of this fact has been presented by Al-Nowaihi et al. [2]. Several results concerning the invariance of the certainty equivalent under the expected utility theory can be found in [3]. The multiattribute utility functions invariant with respect to the various families of transformations have been investigated in [4-7].

The aim of this paper is to generalize the result by Al-Nowaihi et al. twofold. First, we prove that the $\lambda$ homogeneity of the certainty equivalent just for two suitable parameters implies its positive homogeneity. Furthermore, we derive the form of the value function in 
the case where the certainty equivalent is invariant with respect to a more general family of transformations.

\section{Results}

We begin with the results showing that the $\lambda$ homogeneity of the certainty equivalent on the family $\mathcal{X}_{2}$ for two parameters $\lambda$, satisfying some additional assumption, implies its homogeneity on $\mathcal{X}_{2}$.

Theorem 1. Assume that $\lambda_{1}, \lambda_{2} \in(0, \infty) \backslash\{1\}$ and $\ln \lambda_{1} / \ln \lambda_{2}$ is irrational. If the certainty equivalent is $\lambda_{i}-$ homogeneous on $\mathcal{X}_{2}$ for $i \in\{1,2\}$, then the value function is of the form (7) with some $\alpha, \beta, \kappa>0$.

Proof. Assume that the certainty equivalent is $\lambda_{i}$ homogeneous on $\mathcal{X}_{2}$ for $i \in\{1,2\}$. Then, making use of (4) and (6), for every $x>0, p \in[0,1]$ and $i \in\{1,2\}$, we get

$$
\begin{aligned}
& \lambda_{i} u^{-1}(g(p) u(x))=\lambda_{i} C_{u}(x, p)=C_{u}\left(\lambda_{i} x, p\right)= \\
& \quad u^{-1}\left(g(p) u\left(\lambda_{i} x\right)\right) .
\end{aligned}
$$

Replacing in these equalities $x$ by $u^{-1}(y)$, for every $y \in u((0, \infty)), p \in[0,1]$ and $i \in\{1,2\}$, we obtain

$$
\lambda_{i} u^{-1}(g(p) y)=u^{-1}\left(g(p) u\left(\lambda_{i} u^{-1}(y)\right)\right) .
$$

Since $g$ maps $[0,1]$ onto $[0,1]$, this implies that

$$
\begin{aligned}
& u\left(\lambda_{i} u^{-1}(z y)\right)=z u\left(\lambda_{i} u^{-1}(y)\right) \\
& \quad \text { for } y \in u((0, \infty)), z \in[0,1], i \in\{1,2\} .
\end{aligned}
$$

Therefore, for $i \in\{1,2\}$, the functions $f_{i}: u((0, \infty)) \rightarrow$ $\mathbb{R}$, given by

$$
f_{i}(y)=u\left(\lambda_{i} u^{-1}(y)\right) \text { for } y \in u((0, \infty)),
$$

satisfy equation

$$
f_{i}(z y)=z f_{i}(y) \text { for } y \in u((0, \infty)), z \in[0,1] .
$$

Taking an $y_{0} \in u((0, \infty))$ and putting $a_{i}:=\frac{f_{i}\left(y_{0}\right)}{y_{0}}$ for $i \in\{1,2\}$, from (9) we deduce that $f_{i}\left(z y_{0}\right)=a_{i} z y_{0}$ for $z \in[0,1], i \in\{1,2\}$. Hence

$$
f_{i}(x)=a_{i} x \text { for } x \in\left[0, y_{0}\right], i \in\{1,2\} .
$$

Furthermore, taking an arbitrary $y \in u((0, \infty))$, for sufficiently small $z \in(0,1)$, we have $z y \in\left(0, y_{0}\right)$. Therefore, applying (9) and (10), for sufficiently small $z \in(0,1)$, we obtain $z f_{i}(y)=f_{i}(z y)=a_{i} z y$ for $i \in\{1,2\}$. Thus

$$
f_{i}(y)=a_{i} y \text { for } y \in u((0, \infty)), i \in\{1,2\}
$$

and so, taking into account (8), we get

$$
u\left(\lambda_{i} u^{-1}(y)\right)=a_{i} y \text { for } y \in u((0, \infty)), i \in\{1,2\} .
$$

Consequently

$$
u\left(\lambda_{i} x\right)=a_{i} u(x) \text { for } x \in(0, \infty), i \in\{1,2\} .
$$

Since $u(1)=1$, applying (11) with $x=1$, we obtain $u\left(\lambda_{i}\right)=a_{i}$ for $i \in\{1,2\}$. Hence (11) becomes

$$
u\left(\lambda_{i} x\right)=u\left(\lambda_{i}\right) u(x) \text { for } x \in(0, \infty), i \in\{1,2\} .
$$

Therefore, a function $F: \mathbb{R} \rightarrow \mathbb{R}$ given by $F(x)=$ $\ln u\left(e^{x}\right)$ for $x \in \mathbb{R}$, satisfies equation

$$
\begin{aligned}
& F\left(\ln x+\ln \lambda_{i}\right)=F(\ln x)+F\left(\ln \lambda_{i}\right) \\
& \quad \text { for } x \in(0, \infty), i \in\{1,2\},
\end{aligned}
$$

that is

$$
\begin{aligned}
& F\left(x+\ln \lambda_{i}\right)=F(x)+F\left(\ln \lambda_{i}\right) \\
& \quad \text { for } x \in \mathbb{R}, i \in\{1,2\} .
\end{aligned}
$$

Thus, applying Lemma 18.5.1 in [8], we get

$$
F(x+y)=F(x)+F(y) \text { for } x \in \mathbb{R}, y \in A,
$$

where $A$ is a subgroup of the additive group of reals, generated by the set $\left\{\ln \lambda_{1}, \ln \lambda_{2}\right\}$. Moreover, since $\ln \lambda_{1} / \ln \lambda_{2}$ is irrational, $A$ is a dense subset of $\mathbb{R}$. Hence, from (12) it follows that:

$$
F(x+y)=F(x)+F(y) \text { for } x, y \in \mathbb{R} .
$$

Note also that, as $u$ is continuous and strictly increasing, so is $F$. Thus, according to Theorem 5.5.2 in [8], we get $F(x)=\alpha x$ for $x \in \mathbb{R}$ with some $\alpha>0$. Therefore

$$
u(x)=e^{F(\ln x)}=e^{\alpha \ln x}=x^{\alpha} \text { for } x \in(0, \infty) .
$$

The similar arguments lead to

$$
u\left(\lambda_{i} x\right)=a_{i} u(x) \text { for } x \in(-\infty, 0), i \in\{1,2\} .
$$

This implies that

$$
\bar{u}\left(\lambda_{i} x\right)=a_{i} \bar{u}(x) \text { for } x \in(0, \infty), i \in\{1,2\},
$$

where $\bar{u}(x)=u(-x)$ for $x \in(0, \infty)$. Thus $a_{i}=\frac{\bar{u}\left(\lambda_{i}\right)}{\bar{u}(1)}$ for $i \in\{1,2\}$ and so

$$
\frac{\bar{u}\left(\lambda_{i} x\right)}{\bar{u}(1)}=\frac{\bar{u}\left(\lambda_{i}\right)}{\bar{u}(1)} \frac{\bar{u}(x)}{\bar{u}(1)} \text { for } x \in(0, \infty), i \in\{1,2\} .
$$

Therefore, arguing as previously, we conclude that $\bar{u}(x)=\bar{u}(1) x^{\beta}$ for $x \in(0, \infty)$ with some $\beta>0$. Hence $u(x)=-\kappa(-x)^{\beta}$ for $x \in(-\infty, 0)$, where $\kappa:=-\bar{u}(1)=$ $-u(-1)>0$. Therefore, taking into account (13), we obtain (7).

A straightforward calculation shows that if the value function is of the form (7) with some $\alpha, \beta, \kappa>0$, then the certainty equivalent is positively homogeneous on $\mathcal{X}_{2}$. Therefore, from Theorem 1 we derive the following result.

Corollary 1. Assume that $\lambda_{1}, \lambda_{2} \in(0, \infty) \backslash\{1\}$ and $\ln \lambda_{1} / \ln \lambda_{2}$ is irrational. If the certainty equivalent is $\lambda_{i}-$ homogeneous on $\mathcal{X}_{2}$ for $i \in\{1,2\}$, then it is positively homogeneous on $\mathcal{X}_{2}$.

Theorem 2. Let $\lambda_{i} \in(0, \infty) \backslash\{1\}$ for $i \in\{0,1,2\}$ be such that $\ln \lambda_{1} / \ln \lambda_{2}$ is irrational. Assume that the certainty equivalent is $\lambda_{i}$-homogeneous on $\mathcal{X}_{2}$ for $i \in\{1,2\}$ and it is $\lambda_{0}$-homogeneous on $\{L\}$, where $L=\left\langle x_{0}, 1-p_{0} ; y_{0}, p_{0}\right\rangle$ with some $x_{0}<0<y_{0}$ and $p_{0} \in(0,1)$ such that $g\left(p_{0}\right) \neq$ 0 . Then $u$ is of the form (7) with $\alpha=\beta$.

Conversely, if $u$ is of the form (7) with $\alpha=\beta$, then the certainty equivalent is positively homogeneous on $\Delta$.

Proof. According to Theorem 1, $u$ is of the form (7) with some $\alpha, \beta, \kappa>0$. We show that $\alpha=\beta$. In view of (5) and (6), we get

$$
u\left(C_{u}(L)\right)=h\left(1-p_{0}\right) u\left(x_{0}\right)+g\left(p_{0}\right) u\left(y_{0}\right)
$$

and

$$
\begin{aligned}
& u\left(\lambda_{0} C_{u}(L)\right)=u\left(C_{u}\left(\lambda_{0} L\right)\right)=h\left(1-p_{0}\right) u\left(\lambda_{0} x_{0}\right) \\
& \quad+g\left(p_{0}\right) u\left(\lambda_{0} y_{0}\right) .
\end{aligned}
$$


Suppose that $C_{u}(L)<0$. Then, taking into account (7), from the above equalities we derive

and

$$
-\kappa\left(-C_{u}(L)\right)^{\beta}=-\kappa h\left(1-p_{0}\right)\left(-x_{0}\right)^{\beta}+g\left(p_{0}\right) y_{0}^{\alpha}
$$

$$
\begin{aligned}
& -\kappa\left(-\lambda_{0} C_{u}(L)\right)^{\beta}=-\kappa h\left(1-p_{0}\right) \lambda_{0}^{\beta}\left(-x_{0}\right)^{\beta} \\
& \quad+g\left(p_{0}\right) \lambda_{0}^{\alpha} y_{0}^{\alpha},
\end{aligned}
$$

respectively. Hence, multiplying both sides of (14) by $\lambda_{0}^{\beta}$ and subtracting obtained in this way equality from (15), we obtain $\left(\lambda_{0}^{\alpha}-\lambda_{0}^{\beta}\right) g\left(p_{0}\right) y_{0}^{\alpha}=0$. Since $y_{0}>0$ and $g\left(p_{0}\right) \neq 0$, this implies that $\alpha=\beta$. In the case where $C_{u}(L)>0$, the similar arguments work.

In order to prove a converse, assume that $u$ is of the form (7) with $\alpha=\beta$. Then, for every $x \in \mathbb{R}$ and $\lambda>0$, we get $u(\lambda x)=\lambda^{\alpha} u(x)$ and $u^{-1}(\lambda x)=\lambda^{1 / \alpha} u(x)$. Therefore, as the generalized Choquet integral is positively homogeneous, for every $X \in \Delta$ and $\lambda>0$, we obtain

$$
\begin{array}{r}
u^{-1}\left(E_{g h} u(\lambda X)\right)=u^{-1}\left(E_{g h} \lambda^{\alpha} u(X)\right)= \\
u^{-1}\left(\lambda^{\alpha} E_{g h} u(X)\right)=\lambda u^{-1}\left(E_{g h} u(X)\right) .
\end{array}
$$

Hence, in view of (6), $C_{u}$ is positively homogeneous on $\Delta$.

Now, we are going to investigate the invariance of the certainty equivalent with respect to a more general family of transformations. Assume that $u$ is the value function, $I$ is an open real interval and $\Gamma=\left\{\gamma_{t}: t \in I\right\}$ is a given family of transformations, where $\gamma_{t}: \mathbb{R} \rightarrow \mathbb{R}$ for $t \in I$. The certainty equivalent is said to be invariant on $\mathcal{X} \subset \Delta$ with respect to the family $\Gamma$, provided

$$
C_{u}\left(\gamma_{t}(X)\right)=\gamma_{t}\left(C_{u}(X)\right) \text { for } X \in \mathcal{X}, t \in I .
$$

In general, it is not possible to derive the form of the value function from the invariance of the certainty equivalent with respect to $\Gamma$. However, as we will see, it is possible in the case where $\Gamma$ consists of transformations of the form

$$
\gamma_{t}(x)=G^{-1}(d(t) G(x)) \text { for } x \in \mathbb{R}, t \in I,
$$

where $d: I \rightarrow(0, \infty)$ and $G: \mathbb{R} \rightarrow \mathbb{R}$ are given functions such that $G$ is strictly increasing and $G([0, \infty))=[0, \infty)$. In [4] and [7] the multiattribute utility functions with respect to the similar families of transformations have been studied under the expected utility theory. Note that if $I=(0, \infty), G(x)=x$ for $x \in \mathbb{R}$ and $d(t)=t$ for $t \in$ $(0, \infty)$, then $\gamma_{t}(x)=t x$ for $x \in \mathbb{R}$ and $t \in(0, \infty)$. Hence, the invariance of the certainty equivalent with respect to such a family reduces to its positive homogeneity. It is also remarkable that if $\gamma_{t}$ are given by (16), then any two members of $\Gamma$ commute, that is $\gamma_{t} \circ \gamma_{s}=\gamma_{s} \circ \gamma_{t}$ for $s, t \in I$. This means that the impact of applying two members of the family $\Gamma$ does not depend on the order of application. It is remarkable that under some additional assumptions, every family of commuting mappings $\gamma_{t}: I \rightarrow I$ consists of maps of the form (16) with some bijection $G: \mathbb{R} \rightarrow \mathbb{R}$ and $d: I \rightarrow R$. More details concerning this problem can be found in [9] (p. 270-273).

Theorem 3. Let $I \subseteq \mathbb{R}$ be an open interval, $d$ : $I \rightarrow(0, \infty)$ be a nonconstant continuous function and
$G: \mathbb{R} \rightarrow \mathbb{R}$ be a strictly increasing continuous function with $G([0, \infty))=[0, \infty)$. Assume that the certainty equivalent is invariant on $\mathcal{X}_{2}$ with respect to the family $\Gamma=\left\{\gamma_{t}: t \in I\right\}$ of transformations of the form (16). Then the value function is of the form

$$
u(x)= \begin{cases}\delta G(x)^{\alpha} & \text { for } x \geq 0, \\ -\xi[-G(x)]^{\beta} & \text { for } x<0\end{cases}
$$

with some $\alpha, \beta, \delta, \xi>0$.

If, additionally, $C_{u}\left(\gamma_{t_{0}}(L)\right)=\gamma_{t_{0}}\left(C_{u}(L)\right)$ for a $t_{0} \in I$ with $\gamma_{t_{0}} \neq i d_{\mathbb{R}}$ and a lottery $L=\left\langle x_{0}, 1-p_{0} ; y_{0}, p_{0}\right\rangle$ such that $x_{0}<0<y_{0}, p_{0} \in(0,1)$ and $g\left(p_{0}\right) \neq 0$, then we get $\alpha=\beta$ in (17).

Conversely, if $u$ is of the form (17) with some $\alpha, \beta, \delta, \xi>0$, then the certainty equivalent is invariant with respect to the family $\Gamma$ on $\mathcal{X}_{2}$ and, if $\alpha=\beta$, then it is invariant with respect to $\Gamma$ on $\Delta$.

Proof. Since $G$ is strictly increasing and $G([0, \infty))=$ $[0, \infty)$, we have $G(0)=0$. Let $\delta:=\left(u \circ G^{-1}\right)(1)$ and

$$
f:=\frac{1}{\delta}\left(u \circ G^{-1}\right) \text {. }
$$

Note that $\delta>0, f(1)=1$ and $f(0)=0$. Furthermore, as $u$ and $G$ are continuous and strictly increasing, so is $f$. Thus, $f$ is the value function. Fix an $X \in \Delta$, a $t \in I$ and suppose that

$$
C_{u}\left(\gamma_{t}(X)\right)=\gamma_{t}\left(C_{u}(X)\right) .
$$

Thus, taking into account (6) and (16), we obtain

$$
\begin{gathered}
u^{-1}\left(E_{g h} u\left(G^{-1}(d(t) G(X))\right)\right)= \\
G^{-1}\left(d(t) G\left(u^{-1}\left(E_{g h} u(X)\right)\right)\right),
\end{gathered}
$$

which implies that

$$
\begin{aligned}
& \left(G \circ u^{-1}\right)\left(E_{g h}\left(u \circ G^{-1}\right)(d(t) G(X))\right) \\
& =d(t)\left(G \circ u^{-1}\right)\left(E_{g h}\left(u \circ G^{-1}\right)(G(X))\right) .
\end{aligned}
$$

Hence, in view of (6) and (15), we get

$$
C_{f}(d(t) G(X))=d(t) C_{f}(G(X)) .
$$

In this way we have proved that, for every $X \in \Delta$ and $t \in I$, (19) implies (20).

Assume that the certainty equivalent $C_{u}$ is invariant on $\mathcal{X}_{2}$ with respect to the family $\Gamma$. Then (19) holds for every $X \in \mathcal{X}_{2}$ and $t \in I$. Thus (20) is valid for every $X \in \mathcal{X}_{2}$ and $t \in I$. Note also that as $G([0, \infty))=[0, \infty)$, we have $G\left(\mathcal{X}_{2}\right)=\mathcal{X}_{2}$. Therefore, from (20) we derive that $C_{f}(\lambda X)=\lambda C_{f}(X)$ for $X \in \mathcal{X}_{2}$ and $\lambda \in d(I)$. Hence, $C_{f}$ is $\lambda$-homogeneous on $\mathcal{X}_{2}$ for every $\lambda \in d(I)$. On the other hand, as $d$ is continuous and nonconstant, $d(I)$ is a nondegenerate interval. Thus, there exist $\lambda_{1}, \lambda_{2} \in d(I) \backslash\{1\}$ such that $\ln \lambda_{1} / \ln \lambda_{2}$ is irrational. Consequently, applying Theorem 1, we conclude that

$$
f(x)= \begin{cases}x^{\alpha} & \text { for } x \geq 0 \\ -\rho(-x)^{\beta} & \text { for } x<0\end{cases}
$$

with some $\alpha, \beta, \rho>0$. So, taking into account (18), we obtain (17) with $\xi:=\rho \delta>0$.

Assume that $C_{u}\left(\gamma_{t_{0}}(L)\right)=\gamma_{t_{0}}\left(C_{u}(L)\right)$ for a $t_{0} \in I$ such that $\gamma_{t_{0}} \neq \operatorname{id}_{\mathbb{R}}$ and some lottery $L=\left\langle x_{0}, 1-p_{0} ; y_{0}, p_{0}\right\rangle$ with $x_{0}<0<y_{0}, p_{0} \in(0,1)$ and $g\left(p_{0}\right) \neq 0$. Then, as we 
have already proved, (20) holds with $t=t_{0}$ and $X=L$, that is

$$
C_{f}\left(d\left(t_{0}\right) G(L)\right)=d\left(t_{0}\right) C_{f}(G(L)),
$$

where $f$ is given by (18). Moreover, as $\gamma_{t_{0}} \neq \mathrm{id}_{\mathbb{R}}$, in view of $(16)$, we have $d\left(t_{0}\right) \neq 1$. Therefore, since

$$
\gamma_{t_{0}}(L)=\left\langle G^{-1}\left(d(t) G\left(x_{0}\right)\right), 1-p_{0} ; G^{-1}\left(d(t) G\left(y_{0}\right)\right), p_{0}\right\rangle
$$

and $G^{-1}\left(d(t) G\left(x_{0}\right)\right)<0<G^{-1}\left(d(t) G\left(y_{0}\right)\right)$, applying Theorem 2, we get $\alpha=\beta$ in (21) and so in (17).

A straightforward calculation shows that if the value function is of the form (17) with some $\alpha, \beta, \delta, \xi>0$, then the certainty equivalent is invariant with respect to the family $\Gamma$ on $\mathcal{X}_{2}$ and, if $\alpha=\beta$, then it is invariant with respect to $\Gamma$ on $\Delta$.

\section{Conclusion}

We have considered the connections between the invariance of the certainty equivalent under the cumulative prospect theory and the form of the value function. We have proved that a $\lambda$-homogeneity of the certainty equivalent just for two suitable values of the scaling parameter implies its positive homogeneity. Furthermore, we have investigated the invariance of the certainty equivalent with respect to a more general family of transformations. In particular, we have obtained the generalization of the result from the paper by Al-Nowaihi et al. [2].

\section{References}

[1] A. Tversky, D. Kahneman, J. Risk Uncert. 5, 297 (1992).

[2] A. Al-Nowaihi, I. Bradley, S. Dhami, Econ. Lett. 99, 337 (2008).

[3] A.E. Abbas, Decis. Anal. 4, 17 (2007).

[4] A.E. Abbas, Theory Decis. 68, 69 (2010).

[5] A.E. Abbas, J. Aczél, Decis. Anal. 7, 215 (2010).

[6] A.E. Abbas, J. Aczél, J. Chudziak, Result Math. 54, 1 (2009).

[7] J. Chudziak, S. Wójcik, Opuscula Math. 33, 455 (2013).

[8] M. Kuczma, An Introduction to the Theory of Functional Equations and Inequalities, Birkhäuser, Berlin 2009.

[9] J. Aczél, Lectures on functional equations and their applications, Mathematics in Science and Engineering, Academic Press, New York 1966. 